\title{
Die hessische Landtagswahl vom 18. Januar 2009: der „ewige Koch“
}

\author{
Rüdiger Schmitt-Beck und Thorsten Faas
}

Der kürzesten Wahlperiode in der Geschichte Hessens folgte ein kurzer, nicht sehr „knackiger" Wahlkampf, an dessen Ende ein allgemein erwartetes Ergebnis stand: ein klarer Wahlsieg des Bündnisses von CDU und FDP. Nach rasch abgeschlossenen Koalitionsverhandlungen wurde die seit der Landtagswahl im Januar 2008 nur noch geschäftsführend amtierende CDU-Alleinregierung zu einer schwarz-gelben Koalition erweitert (der dritten in Hessen nach 1987 und 1999), die nun anders als bei der Vorwahl über eine bequeme Mehrheit im Landtag verfügt. Ein gutes Jahr nachdem Roland Kochs Ablösung fast gewiss erschien, führte die vorgezogene Neuwahl im Januar 2009 zu seiner klaren Bestätigung im Amt des Ministerpräsidenten, das er seit nunmehr einem vollen Jahrzehnt ununterbrochen im früheren sozialdemokratischen Stammland ausübt. Gravierende Fehler der Sozialdemokraten, die unter Führung von Andrea Ypsilanti in desaströser Weise bei dem Versuch gescheitert waren, den zutiefst abgelehnten CDU-Ministerpräsidenten durch eine von der Linkspartei tolerierte rot-grüne Koalition aus dem Amt zu drängen, führten dazu, dass die SPD ihr im Vorfeld der Landtagswahl 2008 stark gewachsenes Ansehen in der Wählerschaft verspielte und auf ihr schlechtestes Wahlergebnis seit Bestehen des Bundeslandes abstürzte. Nach einer knapp einjährigen Neuauflage der „hessischen Verhältnisse “1 kehrte das Land unter bürgerlichem Vorzeichen zu einer klaren Mehrheit zurück.

\section{Politische Ausgangslage ${ }^{2}$}

Das Ergebnis der Wahl 2008 hatte dem Land eine Neuauflage der „Verhältnisse“ beschert, wie sie ein Vierteljahrhundert zuvor schon einmal kennzeichnend für die hessische Landespolitik gewesen waren: Ebenso wie 1982 und 1983 führte die erstmalige Repräsentanz einer neuen Partei im Landesparlament - damals der Grünen, diesmal der Linkspartei - zu einer Sitzverteilung, auf deren Grundlage keine mehrheitsfähige Regierung gebildet werden konnte. Die traditionelle Polarisierung der hessischen Politik schloss eine Große Koalition

* Die Autoren sind Daniela Hohmann für ihre Unterstützung bei der Materialbeschaffung zu Dank verpflichtet. Dank gilt auch der Forschungsgruppe Wahlen (Mannheim) für die Überlassung des Datensatzes der im Auftrag des ZDF durchgeführten hessischen Vorwahlumfrage zur sekundäranalytischen Auswertung sowie Patrick Kraft für die Unterstützung bei der Aufbereitung dieser Daten. Die Verantwortung für diese Analysen liegt allein bei den Autoren.

1 Vgl. Rüdiger Schmitt-Beck / Thorsten Faas, Die hessische Landtagswahl vom 27. Januar 2008: Wiederkehr der „hessischen Verhältnisse“, in: ZParl, 40. Jg. (2009), H. 1, S. 16 - 34.

2 Basis der Abschnitte 1, 2 und 5 ist die Berichterstattung der Tages- und Wochenpresse, die über die elektronische Datenbank Lexis-Nexis, die Online-Archive der Frankfurter Allgemeinen Zeitung, der Frankfurter Rundschau, der Süddeutschen Zeitung und der Zeit sowie den GoogleNews-Dienst erschlossen wurde. Siehe auch Forschungsgruppe Wahlen, Wahl in Hessen. Eine Analyse der Landtagswahl vom 18. Januar 2009, Berichte der Forschungsgruppe Wahlen e.V., Nr. 133, Mannheim 2009; Infratest dimap, Landtagswahl in Hessen 18. Januar 2009, Berlin 2009. 
aus, lagerübergreifende Dreierverbindungen kamen ebenfalls nicht zustande. Den Regeln der Hessischen Landesverfassung entsprechend war daher die seit 2003 amtierende CDUAlleinregierung geschäftsführend im Amt geblieben. Beflügelt durch ein im Vergleich zu 2003 wesentlich verbessertes Wahlergebnis, das jedoch wegen eines fortbestehenden geringen Rückstandes gegenüber der Union nur zu einem „gefühlten“3, jedoch keinem faktischen Wahlsieg reichte, aber auch durch die Popularität ihrer Spitzenkandidatin Ypsilanti, die im Lande beliebter war als der amtierende Ministerpräsident Koch, entschied sich die SPD für den Versuch, trotz ihrer klaren Ablehnung eines solchen Bündnisses während des Wahlkampfes nun doch eine Kooperation mit der Linkspartei anzustreben. Zwei Versuche, eine von der Linkspartei tolerierte rot-grüne Regierung zu bilden, scheiterten jedoch unter zuletzt dramatischen Umständen schon im Vorfeld, weil einzelne Abgeordnete der SPD diesem Vorhaben die Gefolgschaft verweigerten. Am Ende blieb nur noch der Weg zu Neuwahlen. Einstimmig löste sich der 17. Hessische Landtag am 19. November 2008 nach seiner mit nur 228 Tagen bislang kürzesten Wahlperiode auf. Die Neuwahl wurde auf den 18. Januar 2009 terminiert, und die Parteien begannen umgehend mit den Vorbereitungen ihrer Wahlkampagnen.

An politisches Gestalten war während des kurzen Interregnums kaum zu denken gewesen. Zwar wollte die SPD versuchen, mit Mehrheiten für einzelne Sachanträge aus dem Parlament heraus gegen die Regierung Entscheidungen durchzusetzen, und es gelang auch, wenngleich mit Hindernissen, auf diesem Wege die unpopulären Studiengebühren abzuschaffen. Doch weitere Projekte konnten von den Oppositionsbänken her nicht mehr realisiert werden. Punktuell (bei einem Gesetz zur Beamtenbesoldung) kam es sogar zu einer Kooperation zwischen den Grünen und der CDU. Während es dem Ministerpräsidenten mehrfach gelang, seinen Gegnern Knüppel zwischen die Beine zu werfen, musste er selbst unmittelbar vor der Wahl eine peinliche Niederlage einstecken, als der hessische Verwaltungsgerichtshof urteilte, dass es der Regierung sehr wohl möglich sei, im Zusammenhang mit dem geplanten Ausbau des Frankfurter Flughafens ein Nachtflugverbot zu verfügen etwas, das Koch im letzten Wahlkampf bestritten hatte, um nicht als wortbrüchig zu erscheinen. Auch im landespolitisch hoch kontroversen Politikfeld Bildung sah sich die Regierung mit anhaltendem Unmut von Bürgern und Verbänden konfrontiert.

Insgesamt arbeitete die Zeit jedoch klar für die (geschäftsführend) amtierende Regierung und ihren Chef - woran die SPD selbst erheblichen Anteil hatte. Dass Ypsilanti versuchte, trotz gegenteiliger Erklärungen im Wahlkampf mit den Stimmen der Linkspartei einen Regierungswechsel herbeizuführen, löste massive Entfremdung in der Wählerschaft aus, mit nachfolgender Eintrübung des Ansehens der SPD und ihres Personals auf vielen Dimensionen. Der vor der Landtagswahl 2008 erreichte enorme Reputationsgewinn der Sozialdemokraten und ihrer Spitzenkandidatin wurde so durch eine falsch gewählte politische Strategie in kurzer Zeit wieder verspielt. Schon früh signalisierten die Demoskopen, dass es bei einer Neuwahl für Schwarz-gelb reichen würde, und kurz vor der Wahl bestanden kaum mehr Zweifel, dass die CDU zusammen mit der in den Umfragen stark auftrumpfenden FDP eine „satte Mehrheit“ “ gewinnen würde. Der SPD wurde ein katastrophaler Einbruch prognostiziert; ob die Linkspartei wieder in den Landtag kommen würde, erschien fraglich.

3 Forschungsgruppe Wahlen, a.a.O. (Fn. 2), S. 16.

4 Spiegel Online vom 14. Januar 2009, http://www.spiegel.de/politik/deutschland/0,1518,601188, 00.html. 
Nicht übersehen werden darf bei all dem, dass die Landtagswahl 2009 auch unter Bedingungen einer gravierend veränderten politischen Großwetterlage stattfand, denn die globale Finanz- und beginnende Wirtschaftskrise hatten auch die Parameter der Landespolitik verändert. Die letzte Amtshandlung des Landtages vor seiner Selbstauflösung war denn auch eine einstimmig verabschiedete Erhöhung des Bürgschaftsrahmens des Landes für den angeschlagenen Autohersteller Opel.

\section{Wahlkampf}

Konfrontationen zwischen dem bürgerlichen und dem linken Lager gehörten lange zum Standardrepertoire hessischer Wahlkampagnen, und auch 2009 wurde auf sie nicht verzichtet. Aber insgesamt war es ein „weichgespülter“ Wahlkampf, in dem die Parteien weit weniger als bei der vorangegangenen Wahl auf massive Attacken im Sinne des „negative campaigning" setzten. Auf ein TV-Duell der Konkurrenten um das Amt des hessischen Ministerpräsidenten, wie es im Jahr zuvor erstmals veranstaltet worden war, verzichteten die Parteien diesmal zugunsten einer konventionellen „Elefantenrunde“ aller Parteivorsitzenden. Durch gezielten Einsatz innovativer Kommunikationsformen des Internets vermochte sich der Spitzenkandidat der SPD Thorsten Schäfer-Gümbel besondere Beachtung zu verschaffen.

Zweifellos war es für die SPD nach dem Neuwahlbeschluss schwieriger als für jede andere Partei, eine Linie für ihren Wahlkampf zu definieren. Im Angesicht der drohenden Niederlage musste es ihr primär darum gehen, unter äußerst ungünstigen Umständen die absehbaren Verluste durch Mobilisierung der eigenen Anhänger zu begrenzen; folglich wurde auch offensiv um beide Stimmen geworben. Der gescheiterte Regierungswechsel und seine Umstände sowie der Vorwurf, Wahlversprechen gebrochen zu haben, der sich mit der versuchten Kooperation mit der Linkspartei verband, bedeuteten für die SPD ein beispielloses Debakel, das sie in eine tiefe innerorganisatorische Krise stürzte. Verschwörungstheorien, Schuldzuweisungen, Rücktrittsforderungen und ein Klima von „Angst, Hass und Intrige“ beherrschten die Atmosphäre. Etliche Mitglieder kehrten ihrer Partei den Rücken. Rabiate innerparteiliche Ausgrenzungen und Angriffe gegen die vier Abweichler, die sich dem angestrebten Weg zur Macht verweigert hatten ${ }^{6}$, ließen die Partei ebenfalls nicht im besten Licht erscheinen. In dieser Situation verzichtete die Parteichefin Andrea Ypsilanti, die weithin als Verursacherin des strategischen Fiaskos der SPD gesehen wurde und auch parteiintern dafür Kritik einstecken musste, auf eine erneute Spitzenkandidatur. Sie hielt aber (vorerst) am Partei- und Fraktionsvorsitz fest, was den Willen der Sozialdemokraten zu echter Umorientierung zweifelhaft erscheinen lassen musste. Als Führungsfigur für den Wahlkampf präsentierte die SPD stattdessen den bis dahin kaum bekannten, Ypsilantis Umfeld zugerechneten Landtagsabgeordneten Thorsten Schäfer-Gümbel. Zunächst als Hinterbänkler, „politischer Nobody“ und Marionette Ypsilantis sowie als „Kandidat ohne Chance“7 bespöttelt, vermochte es der aus denkbar unvorteilhafter Konstellation heraus agierende Politiker durch gelungene Auftritte, sich aus dem Schatten Ypsilantis zu emanzipieren und 
allseitigen Respekt samt politischer Statur zu gewinnen. Er gestand personelle und inhaltliche Fehler der Partei ein und entschuldigte sich ausdrücklich für ihren Wortbruch. Er mahnte, dass alle Parteien die „Bunkermentalität“ hinter sich lassen müssten und nahm bekräftigt von der Führung der Bundespartei in Berlin - weitgehenden Abstand von jeglichen (positiven wie negativen) Koalitionsaussagen im Wahlkampf. Außer einer Kooperation unter persönlicher Beteiligung von Roland Koch wurde keine Option ausgeschlossen. Programmatisch sollten die im Vorjahr beschworenen Ziele weiterhin gelten, allerdings mit einer Akzentverschiebung zur Industrie- und Arbeitsmarktpolitik. Ein aktiverer Staat sollte helfen, die Krise zu überwinden, der amtierende Ministerpräsident wurde in diesem Zusammenhang als „Teil des Problems, nicht der Lösung “8 apostrophiert.

Vor dem Hintergrund des - aus ihrer Sicht allein der SPD zuzuschreibenden - Fehlschlages beim Versuch einer Neuauflage von Rot-grün in Hessen suchten die Grünen bei der Neuwahl ihr Heil in größtmöglicher Eigenständigkeit. Erneut geführt von Tarek AlWazir sprachen sie sich gegen die „hessische Krankheit" der „Ausschließeritis“9 aus und hielten sich bewusst alle Koalitionsoptionen offen. Einzig die Durchsetzbarkeit grüner Inhalte sollte den Maßstab für Kooperationsentscheidungen bilden. Beflügelt von guten Werten in den Umfragen versuchten die Grünen, sich in kritischer Absetzung zur sozialdemokratischen „Chaostruppe“10 als verlässliche Partei, gar als „zweite große Kraft in Hessen“ neben der $\mathrm{CDU}^{11}$ zu stilisieren.

Die Linkspartei, erneut mit Willy van Ooyen an der Spitze, verließ sich in ihrer Kampagne, die vor allem das Ziel verfolgte, die gerade errungene Präsenz im Landtag zu verteidigen, auf bewährte Rezepte - in Zeiten der Wirtschaftskrise erst recht: soziale Gerechtigkeit sowie mehr Staat, finanziert durch höhere Besteuerung großer Vermögen. Mehr Aufmerksamkeit erlangte die Linkspartei allerdings durch Presseberichte über interne Querelen; Schlagzeilen machten Streitigkeiten, Mobbing-Vorwürfe, Kritik an „Stasi-Methoden“ und „elitärer Kaderbildung“ sowie spektakuläre Austritte ganzer Ortsverbände sowie prominenter Mitglieder. ${ }^{12}$

Die $C D U$ entschied sich für eine ganz andere Wahlkampfstrategie als im Vorjahr. Sie präsentierte sich diesmal nicht scharf attackierend, sondern moderat. Ihr rasch und in großer Einhelligkeit erneut nominierter Spitzenkandidat Roland Koch gab sich staatsmännisch, geläutert und einsichtsfähig; er räumte ein, dass seine kontroverse, über das Thema Ausländerkriminalität stark emotionalisierende Kampagne von $2008^{13}$ ein Fehler gewesen sei. Auch bezüglich des umstrittenen Themas Bildungspolitik signalisierte er Gesprächsbereitschaft gegenüber seinen Kritikern; unmittelbar vor der Wahl wurde überdies eine milliardenschwere „Ausstattungsinitiative“ für die Schulen auf den Weg gebracht. Zudem legte sich die CDU fest, die Abschaffung der Studiengebühren nicht wieder rückgängig zu machen. Zentrale Themen des Wahlkampfes waren jedoch die heraufziehende Wirtschaftskrise

8 SZ vom 5. Januar 2009.

9 Spiegel Online vom 4. November 2008, http://www.spiegel.de/politik/deutschland/0,1518, 588463,00.html.

10 Spiegel Online vom 10. November 2008, http://www.spiegel.de/politik/deutschland/0,1518, 589571,00.html.

11 Vgl. Frankfurter Rundschau vom 1. Dezember 2008.

12 Forschungsgruppe Wahlen, a.a.O. (Fn. 2), S. 18 f.; Spiegel Online vom 6. Januar 2009, http:// www.spiegel.de/politik/deutschland/0,1518,599791,00.html.

13 Vgl. Rüdiger Schmitt-Beck/ Thorsten Fads, a.a.O. (Fn. 1), S. $19-21$. 
und ihre Bewältigung. „In Zeiten wie diesen“, so die Botschaft der CDU, käme es auf Stabilität, Berechenbarkeit, Kompetenz und Erfahrung an - Motive, die nicht nur die eigenen Stärken in der Wählerwahrnehmung betonen sollten, sondern vor dem Hintergrund der Ereignisse, die zur Neuwahl führten, auch als Stilkritik am politischen Gegner zu lesen waren. Weniger aggressiv im Ton, aber in der Grundkonstellation nicht anders als im Vorjahr, wurde die Landtagswahl auch diesmal zur Lagerentscheidung stilisiert, bei der es darum gehe, ein „rot-rot-grünes Linksbündnis“ zu verhindern und eine stabile bürgerliche Mehrheit zu erkämpfen. Dem korrespondierte eine klare Koalitionsaussage zugunsten der FDP - allerdings verbunden mit dem eindeutigen Signal, dennoch beide Stimmen von den Wählern erhalten zu wollen - und eine ebenso deutliche Absage an jegliche Kooperation mit einer anderen Partei, sei es mit der SPD in einer Großen Koalition oder mit den Grünen in einer „Jamaika“-Koalition. Die Chance, die SPD des Wortbruchs gegenüber dem Wähler zu bezichtigen, ließ Koch nicht verstreichen, ihren Spitzenkandidaten bezeichnete er als „Kühlerfigur“"14 Ypsilantis, die nach wie vor am Steuer der SPD sitze.

In der durch exzellente Umfragewerte genährten Gewissheit eines praktisch sicheren Wahlerfolges trat die FDP im Wahlkampf nur wenig in Erscheinung. Unter Führung von Jörg-Uwe Hahn strebte sie mit klarer Koalitionsaussage in ein Regierungsbündnis mit der CDU, das als eine Art „natürliche Partnerschaft “15 inszeniert wurde. Ihre Positionen waren nur in Nuancen von denen der CDU unterscheidbar.

\section{Wählerstimmung ${ }^{16}$}

Wohl selten hat eine Partei einen hart erarbeiteten, beträchtlichen Ansehensgewinn so rasch und so gründlich verspielt wie die hessische SPD zwischen den Landtagswahlen 2008 und 2009. Wesentliche Ursache hierfür war ihr beharrlich verfolgter Plan, entgegen anders lautender Erklärungen im vorangegangenen Wahlkampf mit der Linkspartei zu kooperieren, um die CDU als Regierungspartei in Wiesbaden abzulösen. Wie die Grünen in ihren Anfangsjahren ${ }^{17}$ befindet sich die Linkspartei unter den Parlamentsparteien in der Position eines Außenseiters. In Hessen fand dies beispielsweise darin seinen Ausdruck, dass eine Mehrheit der Wähler die Präsenz der insgesamt auch sehr negativ eingeschätzten Linkspartei im Landtag ablehnend bewertete. Eine Koalition unter Beteiligung der Linkspartei wurde nur von einer kleinen Minderheit der Wähler befürwortet; die große Mehrheit war dagegen. Auch Andrea Ypsilantis Absicht, sich von der Linkspartei zur Ministerpräsidentin wählen zu lassen, wurde von über 70 Prozent der Wähler abgelehnt; nur 25 Prozent fanden dieses Vorhaben gut. In der Folge wurde das Ansehen der hessischen SPD weit über den unmittelbaren Anlass hinaus beschädigt. Nicht nur ihre allgemeine Wertschätzung in den Augen der Wähler wurde dadurch massiv beeinträchtigt, sondern auch ihre wahrgenommene Glaubwürdigkeit sowie die Einschätzungen ihrer politischen Kompetenz.

14 Zeit Online vom 15. Januar 2009, http://www.zeit.de/online/2009/03/koch-hessen-wahlkampfcdu.

15 SZ vom 17. Januar 2009.

16 Vgl. Forschungsgruppe Wahlen, a.a.O. (Fn. 2), S. 22 - 36; Infratest dimap, a.a.O. (Fn. 2), S. 17 - 48; Viola Neu, Landtagswahlen in Hessen am 18. Januar 2009: Wahlanalyse, Berlin 2009.

17 Vgl. Markus Klein / Jürgen W. Falter, Der lange Weg der Grünen, München 2003, S. 120 - 143. 
Bei vielen Sachfragen büßten die Sozialdemokraten in erheblichem Umfang Kompetenzzuschreibungen in der Wählerschaft ein. In Fragen der Wirtschafts-, Finanz- und Arbeitsmarktpolitik - also gerade in den durch die Wirtschaftskrise direkt berührten und infolge dessen von den Bürgern neben der Bildungspolitik als besonders wichtig erachteten Politikfeldern - fiel sie weit hinter die CDU zurück. In der Familien- sowie der Bildungspolitik und sogar beim Thema der sozialen Gerechtigkeit verschlechterten sich die Kompetenzwahrnehmungen ebenfalls erheblich, auch wenn die SPD hier immer noch vor der Union in Führung lag. Sowohl die allgemeine Befähigung, die Probleme des Bundeslandes zu lösen, bei der 2008 noch die SPD die Nase vorn hatte, als auch die Fähigkeit, die Folgen der Finanz- und Wirtschaftskrise zu bewältigen, sahen klare Mehrheiten der Bürger bei der CDU. Auf der Kandidatendimension ergab sich diesbezüglich kein Gegengewicht, das zugunsten der SPD hätte in die Wagschale fallen können. Vor der Landtagswahl 2008 war Andrea Ypsilanti erheblich populärer als Roland Koch gewesen, aber nach der Wahl verfiel ihr Ansehen rapide; die Mehrzahl der Wähler sah in ihr die Urheberin des Debakels im Landtag. Ihr Nachfolger als Spitzenkandidat wurde zwar in erstaunlich kurzer Zeit weithin bekannt - immerhin etwa 60 Prozent der hessischen Wählerschaft war sein Name direkt vor der Wahl geläufig -, und der Anteil derjenigen, die ihn als künftigen Ministerpräsidenten wünschten, nahm zu. Aber direkt vor der Wahl favorisierten immer noch mehr Wähler den Amtsinhaber als seinen sozialdemokratischen Herausforderer Thorsten Schäfer-Gümbel. $\mathrm{Zu}$ dessen Gunsten sprachen vor allem Beurteilungen „weicher“ Persönlichkeitseigenschaften: Die Wähler fanden ihn im Schnitt sympathischer, glaubwürdiger und ehrlicher als Koch. Die Bewertungen des CDU-Chefs waren vor der Landtagswahl 2009 einmal mehr extrem polarisiert. Sein Image war immer noch von den - in der Krise 2009 freilich gefragten - „harten“ Dimensionen der Tatkraft und Durchsetzungsfähigkeit sowie des Sachverstandes, gerade auch in der Wirtschaftspolitik, geprägt, aber seine persönliche Wertschätzung war gegenüber dem Vorjahr nicht gewachsen. Der insgesamt beliebteste hessische Spitzenpolitiker war der Grüne Tarek Al-Wazir. Anders als die FDP verfügten die Grünen in den Augen der Wähler auch über ein klares Kompetenzprofil; ihnen wurde in erster Linie eine besondere Befähigung in der Umwelt- und Klimapolitik, aber auch in der Energiepolitik zugesprochen.

\section{Wahlergebnis}

\subsection{Das Wahlergebnis in kurz- und langfristiger Perspektive}

Insgesamt boten sich den hessischen Wahlberechtigten bei der Landtagswahl 2009 nur zehn Optionen auf dem Wahlzettel (gegenüber 17 bei der vorangegangenen Wahl): CDU, SPD, FDP, Grüne und Linkspartei zogen in den Hessischen Landtag ein; Freie Wähler, Piraten, NPD, Republikaner und BüSo scheiterten mit einem Stimmenanteil von maximal einem Prozentpunkt allesamt deutlich an der Fünfprozent-Hürde. Die Wahlbeteiligung lag bei 61,0 Prozent (-3,3 Prozentpunkte) - dem niedrigsten Wert der hessischen Nachkriegsgeschichte. Gleichwohl hätte man im Angesicht der „hessischen Verhältnisse“, der damit verbundenen Häufung von Wahlen und des insgesamt wenig spektakulären Wahlkampfes noch Schlimmeres und Geringeres erwarten können. 


\begin{tabular}{|c|c|c|c|c|c|c|c|c|c|}
\hline & \multicolumn{2}{|c|}{$\begin{array}{l}\text { Wahlkreis- } \\
\text { stimmen }\end{array}$} & \multicolumn{4}{|c|}{ Landesstimmen } & \multicolumn{2}{|c|}{ Mandate } & \multirow{3}{*}{$\begin{array}{c}\begin{array}{l}\text { BTW } \\
2005^{3}\end{array} \\
\%\end{array}$} \\
\hline & \multirow[b]{2}{*}{ Anzahl } & \multirow[b]{2}{*}{$\%$} & & & \multicolumn{2}{|c|}{ Veränderung $^{1}$} & \multirow[b]{2}{*}{ Anz. } & \multirow[b]{2}{*}{ Diff. $^{2}$} & \\
\hline & & & Anzahl & $\%$ & Anzahl & \%-Pkt. & & & \\
\hline Wahlberechtigte & 4.375 .286 & & 4.375 .286 & & +4.823 & $+0,1$ & & & \\
\hline Wähler & 2.670 .385 & 61,0 & 2.670 .385 & 61,0 & -140.688 & $-3,3$ & & & 78,7 \\
\hline $\begin{array}{l}\text { Gültige } \\
\text { Stimmen }\end{array}$ & 2.580 .424 & 96,6 & 2.591 .872 & 97,1 & -151.087 & $-0,5$ & & & 97,7 \\
\hline $\mathrm{CDU}$ & 1.083 .174 & 42,0 & 963.763 & 37,2 & -46.012 & $+0,4$ & 46 & +4 & 33,7 \\
\hline SPD & 767.068 & 29,7 & 614.648 & 23,7 & -391.616 & $-13,0$ & 29 & -13 & 35,6 \\
\hline Grüne & 274.492 & 10,6 & 356.040 & 13,7 & +149.430 & $+6,2$ & 17 & +8 & 10,1 \\
\hline FDP & 304.755 & 11,8 & 420.426 & 16,2 & +161.876 & $+6,8$ & 20 & +9 & 11,7 \\
\hline Linke & 117.300 & 4,5 & 139.074 & 5,4 & -1.695 & $+0,3$ & 6 & 0 & 5,3 \\
\hline Sonstige & 33.635 & 1,4 & 97.921 & 3,8 & -23.070 & $-0,7$ & 0 & 0 & 3,6 \\
\hline
\end{tabular}

Hatte bei der vorigen Landtagswahl noch die CDU mit einem Minus von zwölf Prozentpunkten erdrutschartige Verluste hinnehmen müssen, war diesbezüglich bei der Wahl 2009 die SPD an der Reihe: Innerhalb eines Jahres verlor sie dreizehn Prozentpunkte an Unterstützung (was mehr als einem Drittel ihrer Wähler von 2008 entsprach) und erhielt mit 23,7 Prozent weniger als ein Viertel aller abgegebenen gültigen Stimmen - das mit Abstand schlechteste Ergebnis der Sozialdemokraten in der Nachkriegsgeschichte Hessens. Allerdings konnte die CDU unter Koch davon nicht profitieren. Entgegen den Erwartungen stand bei ihr am Ende des Wahlabends ein Verlust von rund 46.000 Landesstimmen in Absolutzahlen zu Buche, der allerdings aufgrund der gesunkenen Wahlbeteiligung trotzdem einem mageren Plus von einem knappen halben Prozentpunkt korrespondierte.

Gewinner der Neuwahl waren die übrigen drei im Landesparlament vertretenen Parteien - Grüne und FDP angesichts ihrer massiven Stimmengewinne, die Linkspartei wegen ihres neuerlichen Einzugs in den Landtag. Mit 16,2 Prozent (und einem Plus von fast sieben Punkten) erzielten die Liberalen ein Traumergebnis (auch wenn sie - wie die langfristige Perspektive zeigt - bei den hessischen Landtagswahlen 1950 und 1954 noch besser hatten abschneiden können); für die Grünen bedeuteten 13,7 Prozent der Stimmen (+ 6,2 Prozentpunkte) das beste Ergebnis, das die Partei jemals in einem Flächenland hat erreichen können. Die Linkspartei konnte ihren Stimmenanteil leicht auf 5,4 Prozent erhöhen (allerdings wie die CDU nur dank der gesunkenen Wahlbeteiligung - absolut büßte sie rund 1.700 Landesstimmen ein). ${ }^{18}$

Im neuen Hessischen Landtag ist die CDU mit 46 Abgeordneten vertreten, die allesamt direkt in einem der 55 Wahlkreise gewählt wurden. Die SPD stellt 29 Abgeordnete (neun

18 Der Wählerwanderung von Infratest dimap zufolge sind die früheren Wähler der SPD vor allem zu den Grünen abgewandert oder erst gar nicht zur Wahl gegangen; die CDU hat vor allem Wähler an die FDP abgegeben (vgl. Infratest dimap, a.a.O. (Fn. 2), S. $12-15$ ). 
davon direkt gewählt), die Liberalen 20, Grüne 17 und Linkspartei sechs. Der neue Landtag besteht somit aus 118 Sitzen - acht mehr als im Normalfall vorgesehen: Der CDU hätten aufgrund ihres Landesstimmenanteils nur 42 Sitze zugestanden, sie erhielt somit vier Überhangmandate. Das hessische Landtagswahlgesetz kennt allerdings auch Ausgleichsmandate, von denen die SPD zwei, Grüne und FDP je eines erhielten.

Setzt man das Wahlergebnis vom 18. Januar 2009 in eine längerfristige Perspektive, zeigen sich bemerkenswerte Ergebnisse: Auf die Tatsachen der niedrigsten Wahlbeteiligung, des schlechtesten SPD- und des besten Grünen-Ergebnisses in der hessischen Geschichte wurde bereits hingewiesen. ${ }^{19}$ Abbildung 1 zeigt darüber hinaus, dass der Grad der Zersplitterung des hessischen Parteiensystems als Ergebnis dieser Wahl höher war als je zuvor. Von einem sehr hohen Wert ausgehend erreichte der Rae-Index als Maß der Fraktionalisierung des Parteiensystems einen bislang in Hessen ungekannten Wert von 0,76, was vor allem dem Wiedereinzug der Linkspartei in den Landtag sowie dem Erstarken von FDP und Grünen geschuldet war. Der Anstieg des Indexes drückt aus, dass sich Hessen einem Vielparteiensystem mit Parteien, die sich hinsichtlich ihrer Stärke in immer geringerem Maße unterscheiden, weiter angenähert hat.

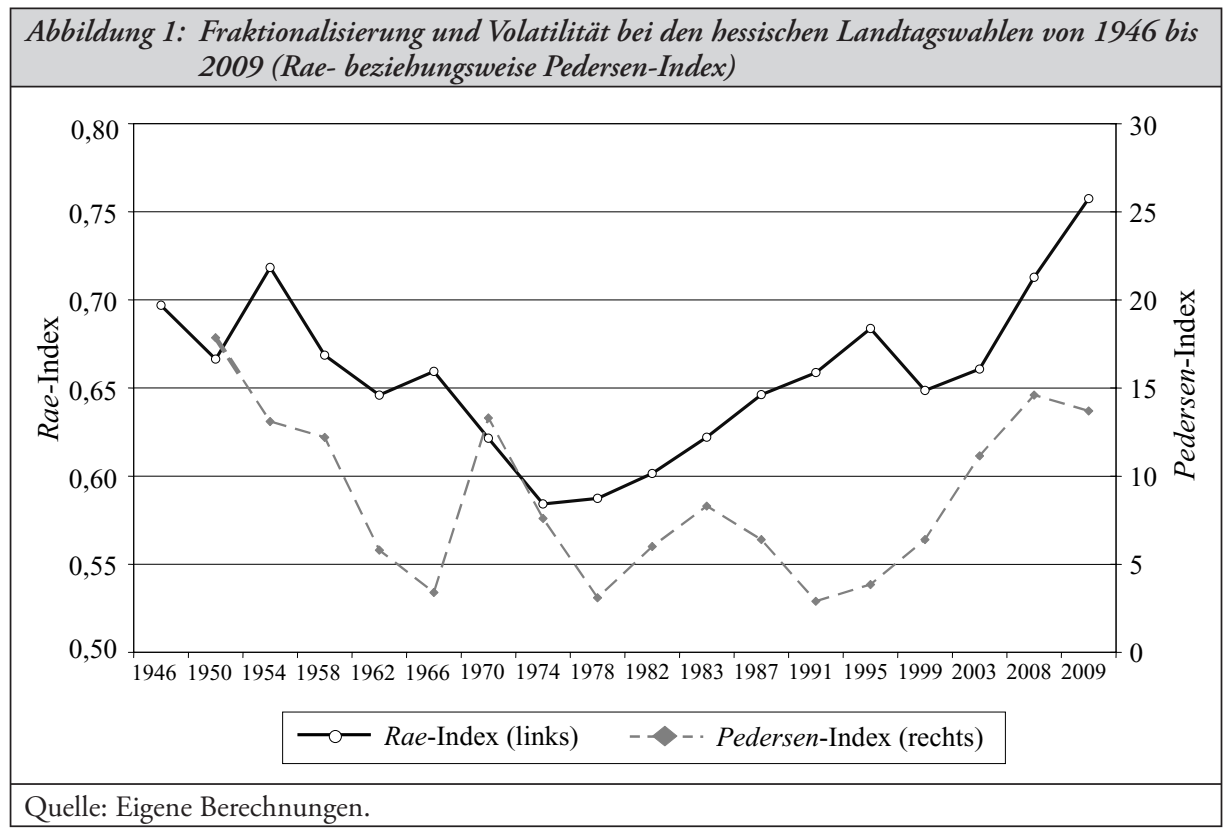

Die elektorale Volatilität hat im Vergleich zu 2008 hingegen nicht weiter zugenommen. Der Pedersen-Index als Aggregatmaß der (Netto-)Wechselhaftigkeit des Wählerverhaltens nahm dieses Mal einen Wert von 13,7 Punkten an. Mindestens 13,7 Prozent der hessischen Wähler haben sich demnach im Vergleich zur vorangegangenen Wahl für eine andere Partei entschieden, was schon alleine durch die dramatischen Verluste der SPD begründet ist. Die Wahl 2008 war allerdings schon durch ähnlich hohe Verluste auf Seiten der CDU (sowie

19 Auch die Linkspartei erzielte ihr bisher bestes Ergebnis, trat allerdings erst zum zweiten Mal an. 
weitere Einbußen der Grünen) gekennzeichnet gewesen und hatte somit zu einer erheblichen Steigerung der Volatilität geführt; diese pendelte sich bei der Neuwahl auf demselben hohen (nur 1950 übertroffenen) Niveau ein.

\subsection{Hintergründe des Wahlergebnisses}

Die Analyse der Hintergründe des Wahlverhaltens bei der Landtagswahl $2008^{20}$ hatte gezeigt, dass sich damals - neben einem stark ausgeprägten, von Koch und Ypsilanti ausgehenden Kandidateneffekt - vor allem die Bildungspolitik zu Gunsten der SPD (und zu Ungunsten der CDU) ausgewirkt hatte, während die von den beiden Parteien selbst im Laufe des Wahlkampfs in den Vordergrund gerückten Themen (soziale Gerechtigkeit / Mindestlohn beziehungsweise Jugend- und Ausländerkriminalität) kaum eine Rolle spielten.

Welche Themeneinflüsse lassen sich für die hessische Landtagswahl 2009 ausmachen? Tabelle 2 zeigt die Ergebnisse von Modellen zur Erklärung der Wahlentscheidungen 2009. Diese berücksichtigen neben den Effekten von Parteibindungen, Kandidatenpräferenzen und der Wichtigkeit politischer Sachfragen auch - den besonderen Umständen dieser Wahl geschuldet - das Thema der Neuwahl selbst, um zu ermitteln, ob es sich im Wahlverhalten niederschlug, wenn Wähler die Neuwahl selbst als ein Problem sahen. ${ }^{21}$ Die Ergebnisse zeigen (der wahlsoziologischen Orthodoxie entsprechend), dass diejenigen Personen, die sich mit einer Partei identifizierten, diese auch mit hoher Wahrscheinlichkeit wählten. Auch von der Präferenz für Koch oder Schäfer-Gümbel als zukünftigen Ministerpräsidenten gingen Effekte auf das Wahlverhalten aus. Koch zu präferieren ging einher mit einer höheren Wahrscheinlichkeit, CDU zu wählen; analog führte eine Präferenz für Schäfer-Gümbel zu einer verstärkten Neigung, für die SPD zu stimmen. ${ }^{22}$ Ebenso bedeutsam aber waren die negativen Effekte: Koch nicht zu mögen, ging einher mit einer höheren Wahrscheinlichkeit, für SPD, Grüne oder die Linkspartei zu votieren, wobei der Effekt bei den Wählern der Grünen am stärksten ausfiel. Analog galt allerdings auch hier: Schäfer-Gümbel nicht zu mögen ging einher mit einer höheren Wahrscheinlichkeit der Wahl von CDU oder FDP. Positiv wirkten sich die Kandidaten demnach nur bei den Anhängern der eigenen Partei aus, die negativen Effekte dagegen strahlten ins gesamte gegnerische Lager aus.

Was die thematischen Prioritäten der Wähler betrifft, so erwies sich die Bildungspolitik auch 2009 noch als prägend, jedoch in ganz anderer Weise als bei der Wahl 2008. Damals hatte das Thema noch eindeutig zu Gunsten der SPD gewirkt; dieser Effekt verpuffte innerhalb eines Jahres: Wer 2009 die Bildungspolitik für ein wichtiges Problem hielt, wandte sich von CDU und SPD ab und den Grünen zu. Ein ähnliches Bild zeigt sich bezüglich der Klima- und Energiepolitik: Auch von diesem Thema konnten die Sozialdemokraten 2008 noch (zu Lasten der CDU) profitieren, während es bei der Neuauflage der Wahl den Grü-

20 Vgl. Rüdiger Schmitt-Beck/ Thorsten Faas, a.a.O. (Fn. 1), S. 29 - 31.

21 Es fehlte im Unterschied zu 2008 das Thema „(Jugend-)Kriminalität“: Zu wenige Bürger nannten 2009 dieses Thema als wichtiges, um es in die Analyse aufnehmen zu können.

22 In einem hier nicht tabellarisch ausgewiesenen Modell, das demjenigen nachgebildet ist, das wir für die Landtagswahl 2008 geschätzt hatten (vgl. Rüdiger Schmitt-Beck / Thorsten Faas, a.a.O. (Fn. 1), S. 29 - 31), zeigte sich, dass die Kandidateneffekte 2008 und 2009 trotz des Wechsels in der sozialdemokratischen Spitzenkandidatur in ähnlicher Größenordnung lagen. 
nen nützte. Die Linkspartei erschien 2009 als Single-Issue-Partei und wurde lediglich von Wählern, die das Thema „Soziale Gerechtigkeit“ als dringlich empfanden, verstärkt unterstützt. Neben den standardmäßigen Erklärungsgrößen - Parteibindungen, Kandidatenpräferenzen und sachpolitischen Orientierungen ${ }^{23}$ - fanden aber auch die besonderen Umstände der Landtagswahl 2009 einen Niederschlag im Wahlverhalten. Wer die Neuwahl selbst für ein wichtiges politisches Problem hielt, der wandte sich von den Sozialdemokraten $\mathrm{ab}$, aber in geringerem Ausmaß - und dies durchaus überraschend - auch von der Union. ${ }^{24}$ Klare Nutznießer dieses Themas waren Liberale und Grüne.

\begin{tabular}{|c|c|c|c|c|c|}
\hline \multicolumn{6}{|c|}{$\begin{aligned} \text { Tabelle 2: Hintergründe der Wablentscheidungen bei der hessischen Landtagswabl } 2009 \\
\text { (b-Koeffizienten logistischer Regressionsmodelle) }\end{aligned}$} \\
\hline & \multicolumn{5}{|c|}{ Gewählte Partei } \\
\hline & $\mathrm{CDU}$ & SPD & FDP & Grüne & Linkspartei \\
\hline $\begin{array}{l}\text { Parteiidentifikation zugunsten } \\
\text { der jeweiligen Partei }\end{array}$ & $2,350^{* * *}$ & $2,727^{* * *}$ & $3,651^{* * *}$ & $3,631^{* * *}$ & $4,259^{* * *}$ \\
\hline \multicolumn{6}{|l|}{ Präferenz Ministerpräsident: } \\
\hline Koch & $1,122^{* * *}$ & $-1,549^{* * *}$ & $-0,309$ & $-2,055^{* * *}$ & $-1,388^{*}$ \\
\hline Schäfer-Gümbel & $-1,949^{* * *}$ & $1,584^{* * *}$ & $-1,713^{* * *}$ & $-0,084$ & 0,343 \\
\hline \multicolumn{6}{|c|}{ Wichtigkeit von Wahlkampfthemen: } \\
\hline Bildung & $-0,703^{* * *}$ & $-0,696^{* *}$ & 0,181 & $1,062^{* * *}$ & 0,236 \\
\hline Wirtschaft / Arbeitslosigkeit & $-0,100$ & $-0,052$ & 0,002 & $-0,000$ & $-0,170$ \\
\hline Soziale Gerechtigkeit & $-0,209$ & $-0,972$ & $-0,401$ & $-0,607$ & $1,856^{* * *}$ \\
\hline Energie- / Klimapolitik & $-1,061^{*}$ & $-0,540$ & 0,246 & $0,828^{*}$ & 0,253 \\
\hline Flughafen Frankfurt & 0,511 & $-0,513$ & $-0,122$ & 0,378 & - \\
\hline Neuwahl Hessen & $-0,496^{\#}$ & $-0,909^{* *}$ & $0,539^{*}$ & $0,893^{* *}$ & 0,167 \\
\hline Konstante & $-0,955^{* * *}$ & $-2,259^{* * *}$ & $-1,527^{* * *}$ & $-2,386^{* * *}$ & $-3,339^{* * *}$ \\
\hline $\mathrm{N}$ & 1080 & 1080 & 1080 & 1080 & 997 \\
\hline (Pseudo-) $\mathrm{R}^{2}$ & 0,626 & 0,611 & 0,284 & 0,472 & 0,278 \\
\hline \multicolumn{6}{|c|}{$\begin{array}{l}\text { Hinweise: \# } \mathrm{p}<0,1 ;{ }^{*} \mathrm{p}<0,05 ;{ }^{* *} \mathrm{p}<0,01 ;{ }^{* * *} \mathrm{p}<0,001 \text {. } \\
\text { Abhängige Variablen: Wahlentscheidung zugunsten der jeweiligen Partei (1); Befragte, die eine andere } \\
\text { Partei wählen wollen, erhalten den Wert 0, Befragte, die keine Partei nennen oder nicht zur Wahl gehen, } \\
\text { bleiben ausgeschlossen. } \\
\text { Unabhängige Variablen: Identifikation mit der jeweiligen Partei (1). Befragte, die sich mit einer anderen } \\
\text { oder keiner Partei identifizieren, erhalten den Wert 0. Präferenz Ministerpräsident Koch oder Schäfer- } \\
\text { Gümbel: Die Referenzkategorie sind Befragte ohne Präferenz für einen der beiden. Wichtigkeit von Wahl- } \\
\text { kampfthemen jeweils: Problem genannt (1), Problem nicht genannt }(0) \text {. } \\
\text { Quelle: Vorwahlbefragung der Forschungsgruppe Wahlen, } \mathrm{n}=1.539 \text {, eigene Auswertungen. }\end{array}$} \\
\hline
\end{tabular}

Insgesamt zeigt die Analyse deutlich, dass das Desaster der misslungenen Regierungsbildung zum Desaster der SPD am Wahlabend geführt hat: Alle Vorteile, die sich die Sozialdemokraten im Vorfeld der Wahl 2008 (etwa auf den Gebieten der Bildungs- und Klima-

23 Vgl. zum Beispiel Jürgen W. Falter / Harald Schoen, Handbuch Wahlforschung, Wiesbaden 2005, S. $187-242$.

24 Für die SPD ließ sich in weitergehenden Analysen (in deren Rahmen die Berechnungsbasis auf frühere SPD-Wähler beschränkt wird) auch zeigen, dass sich viele dieser Wähler von ihr abgewandt haben, sofern sie das Thema "Neuwahl“ als Problem benannten. 
politik) erarbeitet hatten, haben sich innerhalb eines Jahres ins Gegenteil verkehrt ${ }^{25}$; hinzu kam ein starker negativer Effekt des Themas „Neuwahl“ selbst. Profitieren konnte hiervon allerdings weniger die CDU, sondern vor allem FDP und Grüne.

\section{Regierungs- und Oppositionsbildung}

Wie schon vor der Wahl angekündigt, nahmen CDU und FDP umgehend Koalitionsverhandlungen auf und führten diese in kurzer Zeit zu einem einvernehmlichen Abschluss. Innerhalb von zwei Wochen wurde unter dem Titel „Vertrauen. Freiheit. Fortschritt.“ eine Koalitionsvereinbarung erarbeitet, die insbesondere in der Schulpolitik neue Zielakzente zu setzen versuchte, etwa durch eine Aufstockung des Personals, kleinere Klassen und die Option für Schulen, eigenverantwortlicher zu agieren. Bei gleichbleibendem Gesamtumfang des öffentlichen Dienstes wurden auch neue Stellen für die Polizei vorgesehen. Als langfristiges Projekt, das eine Volksabstimmung erfordern würde, nahm sich die neue schwarzgelbe Koalition vor, in der Landesverfassung ein Schuldenverbot zu verankern. Wegen ihres guten Wahlergebnisses konnte die FDP drei von insgesamt zehn Ministerposten besetzen (siehe Tabelle 3). Das Ressort für Justiz, Integration und Europaangelegenheiten übernahm gemeinsam mit dem Posten des stellvertretenden Ministerpräsidenten Jörg-Uwe Hahn. Ebenfalls den Liberalen zugesprochen wurden das Ministerium für Wirtschaft und Verkehr sowie das Kultusministerium. Wie vorgesehen wurde Roland Koch schon bei der konstituierenden Sitzung des Landtages am 5. Februar 2009 erneut zum Ministerpräsidenten des Landes Hessen gewählt, allerdings nur mit 62 Stimmen: Mindestens vier Abgeordnete aus den Reihen von CDU oder FDP haben ihm folglich die Unterstützung verweigert.

\begin{tabular}{|l|l|}
\hline Tabelle 3: Das Kabinett nach der hessischen Landtagswahl vom 18. Januar 2009 \\
\hline Ministerpräsident & Roland Koch (CDU) \\
\hline $\begin{array}{l}\text { Minister der Justiz, für Integration und Europa } \\
\text { (zugleich stellvertretender Ministerpräsident) }\end{array}$ & Jörg-Uwe Hahn (FDP) \\
\hline Minister für Wirtschaft, Verkehr und Landesentwicklung & Dieter Posch (FDP) \\
\hline Kultusministerin & Dorothea Henzler (FDP) \\
\hline Ministerin für Wissenschaft und Kunst & Eva Kühne-Hörmann (CDU) \\
\hline Minister für Arbeit, Familie und Gesundheit & Jürgen Banzer (CDU) \\
\hline Minister der Finanzen & Karlheinz Weimar (CDU) \\
\hline $\begin{array}{l}\text { Minister für Bundes- und Europaangelegenheiten und } \\
\text { Bevollmächtigter des Landes Hessen beim Bund }\end{array}$ & Michael Boddenberg (CDU) \\
\hline Ministerin für Umwelt, Energie, Landwirtschaft und Verbraucherschutz & Silke Lautenschläger (CDU) \\
\hline Staatsminister, Chef der Staatskanzlei & Stefan Grüttner (CDU) \\
\hline Minister des Innern und für Sport & Volker Bouffer (CDU) \\
\hline $\begin{array}{l}\text { Quelle: Landesinformationssystem des Hessischen Landtages, Plenarprotokoll 18/1 vom 5. Februar } \\
\text { 2009, S.18 f. }\end{array}$ \\
\hline
\end{tabular}

25 Dies galt auch mit Blick auf sozialstrukturelle Gruppen: 2008 konnte die SPD vor allem in der Gruppe der formal hoch Gebildeten Zugewinne verbuchen; genau diese Gruppe hat sich 2009 verstärkt von ihr ab- und den Grünen zugewandt. 
Als wichtige bundespolitische Implikation folgte aus der Regierungsbildung eine Machtverschiebung im Bundesrat. Durch den Wiedereinstieg der Liberalen in die Wiesbadener Regierung kann die Große Koalition in Berlin aus CDU/CSU und SPD nicht mehr auf die Stimmen Hessens zählen. Da die verbleibenden Länder, in denen SPD oder Union entweder allein oder aber gemeinsam regieren, nur noch über 30 (statt zuvor 35) der 69 Stimmen verfügen, ist die Zeit einer stabilen Bundesratsmehrheit der Großen Koalition vorbei. Der umgehende Vorstoß der FDP, dadurch Einfluss auf das zweite Konjunkturpaket der Bundesregierung zu gewinnen, scheiterte allerdings daran, dass zwei Landesregierungen, an denen die Grünen beteiligt waren, der Großen Koalition zur Seite sprangen.

Auf Seiten der SPD wurde eine Neuorientierung der Partei eingeleitet. Nachdem Andrea Ypsilanti die politische Verantwortung für das Wahlergebnis übernommen hatte und vom Fraktions- wie Parteivorsitz zurückgetreten war, folgte ihr Thorsten Schäfer-Gümbel in beiden Ämtern nach. Auf sein Betreiben lösten sich die beiden organisierten Flügel innerhalb der SPD-Fraktion - der „Vorwärts“-Kreis der Parteilinken und die wirtschaftsfreundlichere „Aufwärts“-Gruppe - auf.

\section{Zusammenfassung}

(1) Als vorgezogene Neuwahl folgte die hessische Landtagswahl 2009 weniger als ein Jahr auf den vorangegangenen, regulären Wahlgang. 2008 hatte die Linkspartei den erstmaligen Einzug in den Landtag geschafft, in dem dadurch ein parlamentarisches FünfParteien-System mit einer Sitzverteilung entstand, in deren Folge es den Parteien nicht gelang, eine mehrheitsfähige Regierung zu bilden. Die seit 2003 amtierende CDU-Alleinregierung blieb daher geschäftsführend im Amt. Die Neuwahl wurde notwendig, nachdem Versuche der SPD, eine von der Linkspartei tolerierte rot-grüne Regierung zu bilden, an mangelnder Gefolgschaft in den eigenen Reihen gescheitert waren. Wichtig für die Wahl war aber auch die gravierend veränderte politische Großwetterlage infolge der globalen Finanz- und beginnenden Wirtschaftskrise.

(2) Wie bei hessischen Landtagswahlen üblich, wurde auch der Wahlkampf 2009 von den großen Parteien als Konfrontation zwischen dem bürgerlichen und dem linken Lager inszeniert. Aber insgesamt war es ein „weichgespülter“ Wahlkampf, in dem die Parteien weit weniger als bei der Vorwahl auf „negative campaigning“ setzten. CDU und FDP strebten auf der Basis wechselseitiger Koalitionsaussagen und gestützt durch vorteilhafte Umfrageergebnisse nach einer Wiederaufnahme der schwarz-gelben Zusammenarbeit unter dem bisherigen Ministerpräsidenten Roland Koch. Die SPD trat mit dem vorher weitgehend unbekannten Landtagsabgeordneten Thorsten Schäfer-Gümbel an und verfolgte in erster Linie das Ziel der Schadensbegrenzung. Die Spitzenkandidatin der vorangegangenen Wahl, Andrea Ypsilanti, der vielfach die Verantwortung für das Debakel der SPD angelastet wurde, das zur Neuwahl führte, blieb jedoch weiterhin Partei- und Fraktionsvorsitzende; erst nach der Wahl gab sie auch diese Ämter auf. Wie die SPD verzichteten auch die Grünen auf Koalitionsaussagen; sie verfolgten im Wahlkampf einen Kurs betonter Eigenständigkeit. Der Wahlkampf der Linkspartei wurde von innerparteilichen Querelen belastet.

(3) Der beachtliche Ansehensgewinn, den die SPD im Vorfeld der Landtagswahl 2008 hatte erreichen können, ging danach in kurzer Zeit durch den missglückten Versuch der 
Regierungsübernahme und seine Umstände wieder verloren. Im Hinblick auf ihre allgemeine Wertschätzung sowie ihre wahrgenommene Glaubwürdigkeit und Problemlösungskompetenz ging die SPD mit ungünstigen Werten in den Wahlkampf. Der Spitzenkandidat konnte das nicht ausgleichen; zwar genoss Koch bei den Wählern keine höhere Wertschätzung als 2008, aber in der Kürze der verfügbaren Zeit konnte SchäferGümbel keine größere Popularität aufbauen. Die Meinungsumfragen ließen übereinstimmend eine klare Mehrheit für Schwarz-gelb und eine gravierende Niederlage für die Sozialdemokraten erwarten.

(4) Dem Desaster der misslungenen Regierungsbildung folgte für die SPD ein Desaster am Wahlabend, von dem aber nicht die Union, sondern FDP und Grüne profitieren konnten; auch der Linkspartei gelang der Wiedereinzug in den Landtag. Bei leicht gesunkener Wahlbeteiligung führte der Urnengang insgesamt zu einem Hessischen Landtag, der (gemessen über entsprechende Fraktionalisierungsindizes) zersplitterter ist denn je. Analysen der Hintergründe des Wahlergebnisses zeigen, dass der SPD alle Vorteile, die sich die Partei im Vorfeld der Wahl 2008 (etwa auf den Gebieten der Bildungs- und Klimapolitik, aber auch in der Gruppe der formal hoch gebildeten Wähler) erarbeitet hatte, wieder verloren gegangen waren. Hinzu kam ein starker negativer Effekt des Themas „Neuwahl“ selbst, der ebenfalls zu einer Abkehr vor allem von den Sozialdemokraten, aber auch von der CDU führte, die FDP und die Grünen jedoch stärkte.

(5) Nach raschen Koalitionsverhandlungen vereinbarten CDU und FDP ihre dritte Zusammenarbeit in Hessen. Roland Koch wurde wieder zum Ministerpräsidenten gewählt, wenngleich nicht mit allen Stimmen seiner Koalition. Als wichtige bundespolitische Implikation folgte aus der Bildung der CDU-FDP Regierung, dass die Große Koalition in Berlin künftig im Bundesrat keine eigene Mehrheit mehr hat, sondern auf die Zustimmung von Landesregierungen mit Beteiligung anderer Parteien angewiesen ist.

\title{
Parteimitgliedschaften im Jahre 2008
}

\author{
Oskar Niedermayer
}

Mit der folgenden Dokumentation wird der seit 2001 jährlich erscheinende systematische Überblick über die Entwicklung, regionale Verteilung und sozialstrukturelle Zusammensetzung der Parteimitgliedschaften fortgeschrieben. Zusätzliche Daten beziehungsweise Schaubilder sind über das Internet abrufbar (http://www.polsoz.fu-berlin.de/polwiss/forschung/ systeme/empsoz/schriften/Arbeitshefte/ahosz15.pdf). ${ }^{1}$ Es werden nur Daten aus den Mit-

1 Für eine Analyse dieser Daten sowie des Zeitraums vor der Vereinigung vgl. Oskar Niedermayer, Der Wandel des parteipolitischen Engagements der Bürger, in: Steffen Kühnel / ders. / Bettina Westle (Hrsg.), Wähler in Deutschland. Sozialer und politischer Wandel, Gender und Wahlverhalten, Wiesbaden 2008. 